\title{
Methods of Visual Inspection and Application in Flight
}

\author{
Song $\mathrm{Li}^{1, \mathrm{a}^{*}}$, Yuankai $\mathrm{Li}^{1, \mathrm{~b}}$, Xiujuan $\mathrm{Liu}^{1, \mathrm{c}}$ and Limin Song ${ }^{1, \mathrm{~d}}$ \\ ${ }^{1}$ Air Force Aviation University, Changchun, China \\ alisong4885@126.com, byuankaili@126.com, cxiujuanliu@163.com, diminsong@sina.com
}

Keywords: visual inspection; eye movements; flight; attention allocation

\begin{abstract}
Visual inspection is a very important part of the flight, also is the basic skills possessed by the pilot. This paper introduces the concept of visual inspection, summarizes the basic methods of visual inspection and development and research status of eye-tracking test technology at home and abroad, points out the eye movement technology in the application of testing visual inspection skill and directing flight training. Combining with the flight example, the method of visual flight is illustrated and effect of eye movement technology on improving flight training is demonstrated.
\end{abstract}

\section{Introduction}

The airplane driving is a complex task, highly dependent on the visual information effectively to obtain reasonable allocation and attention. With the improvement of aircraft performance and function and increase in cockpit display information, therefore, correct understanding of the pilot's visual scanning behavior to get effective information about the distribution of attention, become more and more experts concern and research hot spot.

Flight is often mentioned in "think, see, touch, check". Although "want to" dominated by brain, do not need to be directly involved in the vision and "move" is the brain control body directly, but these two are involved in visual perception behavior. As to "see" and "check", they are the embodiment of visual behavior. So, the ability of visual behavior has become an important restriction factor affecting the airplane driving skills.

Relevant Concepts of Visual Inspection. Visual inspection refers to use eyes to estimate the distance, the height and angle of approach without the instrument, measurement to the unaided eye, actually this is a military ranging method. Pilot's visual information perception behavior describes the driver under visual flight environment and aircraft information to the brain process motion state, etc.

Note: due to the flight air environment is complex, the pilots through visual can get a lot of information, but the information is not to affect the current driving behavior. In general, the pilot just focus on selectively accepted the most useful to flight information, and tries to process accordingly, other information on the back of the pilots driving operation to be considered or ignored directly.

Information processing is an important condition to ensure flight safety for the pilots. Pilots in commonly found outside stimuli, can make right judgment within $0.5 \sim \mathrm{l}$ s, and adopt corresponding measures to adjust the driving operation, so as to change the state of airplane motion.

During flight, the pilot shift gaze from one target to another target, and in the brain is still retains a view of the target before, this is the information storage. Information storage is actually finished after the stimulus was to stay. Information storage is also known as memory.

According to pilot's experience, knowledge and stimulation (information) of certain features, judgment analysis was carried out on the input information. That is the process of information processing. After the information processing, it is concluded that a corresponding to the external environment of decision, the decision information through man's effect on organ function form, make the airplane according to the scheduled track run. The pilot's information processing is throughout the whole flight process. Obviously, if deal with the outside information improperly, it will make wrong decisions, resulting in the happening of the accident.

In addition, data show that in the process of the pilot to fly a airplane, 95\% of the visual information is dynamic, and is the most closely related in the flight dynamic visual characteristics of 
the pilot, the pilot in the motion state observing things, its visual characteristics is significantly different with the stationary state, for example, reduced visual sharpness, narrow field of vision and space recognition range and so on.

Flight path and the airplane motion of decision-making behavior describes the pilot's processing to obtain information about driving and analyses the ideals of the movement of the control airplane to decision-making instructions brain thinking activity. According to the requirements of the mission, the driver will be the first to predict the future form of exercise and over a period of time after the airplane likely reach the location and status. The location and movement state and may not be accurate, but basically can describe the movement of the airplane, and then according to its prediction to consider the influence of the factors from the feasible expected trajectory point to select the ideal expected trajectory points as the moment the airplane the desired trajectory.

In decisions out of the ideal expected trajectory, the pilot can decision of the ideal operation instruction, but the lagging behind of the pilot and the aircraft itself characteristics, the actual driving action generated by the command is not exactly the same as that of the ideal motion commands. Pilots fly through long-term practice and understanding of driving airplane and adaptation to airplane, motion commands can be treated in a certain correction, to produce the desired action. Pilot operation correction is described pilots according to the previous decisions of the desired trajectory, by considering the flight dynamics characteristics, and the hysteresis characteristics of pilot itself these factors, the decision of the desired trajectory to a certain correction and make practical action in this process[1].

Basic methods of visual inspection. The basic forms of eye movement are watching, twitching, and following. For the most periods of flight, the pilot's eye movement amplitude is less than 6 degrees. The duration of eye fixed on the target is for $100 \sim 350 \mathrm{~ms}$ and within about four degrees $90 \%$ of eye location in the center of the visual range. As the growth of the experience, the driver's eye movement has a significant change, mainly reflected in space and time of changes became very small.

New pilot's visual search is desultorily, eye movement range is larger, even will focus on some irrelevant scenery. After a period of time, the eye movement gradually become stable, alternately at a nearby and distant, has certain regularity. Experienced pilots at a distance of linear search, most of the time looking at nearby of search time is less, while the new pilots at a distance and look at the near time. Experienced pilots are looking at a distance, with the distance of the information have ample time to prepare, at the same time with peripheral retinal visual observation near things. And new pilots worry about its own aircraft track, so put the fixation point nearby.

According to the characteristics of the pilot eye movements and laws, we can use the following methods to evaluate the pilot's visual skills [1].

First, pilots can track the change of eye gaze, so that more visually describe the process of eye movements. It reflects the instantaneous position of pilot fixation point. Second, you can examine the fixed area at the time, to reflect a fixed area and the level of concern of the pilot. Third, investigate the distribution of fixation time, namely at a particular time period, look at the percentage to the total time. This approach can intuitively reflect the monitoring frequency on a particular area in the whole flight process. The final is the angular velocity of the eye movements. Normally, when the pilot in observing a certain important target objects or handle some highlight information, eyes angular velocity will have substantial changes.

Eye-tracking test technology. At present, the commonly used method of visual inspection is eye tracking technology. The main visual tracking technology at home and abroad has the following several kinds, that is Electro-oculogram, Contact Lens, Electromagnetic Coil, Corneal reflection, Infrared Phototelectric and Infrared TV[2].

The Smart Eye non-invasive Eye movement instrument can identify the line of sight direction through the corneal reflection and head model. Figure 1 shows the hardware assembly using 2 3 camera system. 


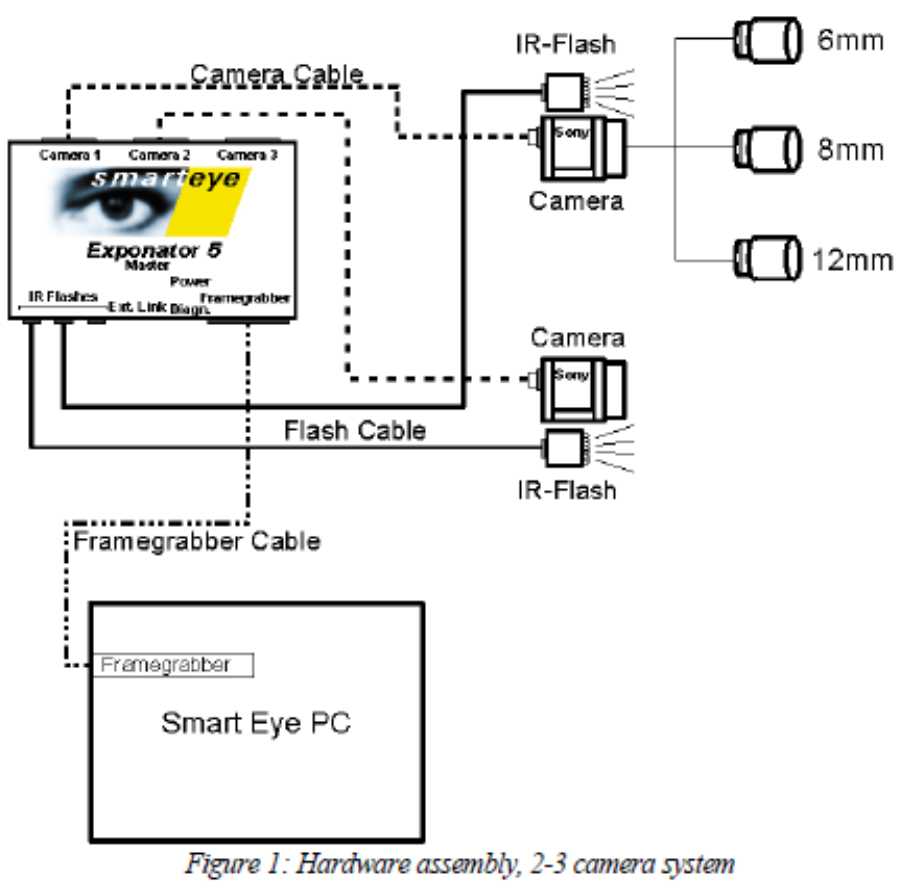

Fig. 1 The hardware assemble of Smart Eye non-invasive Eye movement instrument

Application of testing visual inspection skill and direction to the flight training. All kinds of advanced instrument and equipment provided the motion parameters of aircraft, flight trajectory and the information such as cockpit inside and outside environment, all of these need to be collected and processed by a pilot in a short period of time through the visual inspection, and in some special flight phase (e.g., take-off and landing phases), also need the pilots rely on visual analysis by the naked eye, this put forward higher requirements to the transfer of the pilot's attention and distribution capacity.

Pilots in the process of the airplane take-off, need to get a lot of information, observation in the dashboard can gain speed, rate of climb, heading and altitude data, observation tank location can ensure that in the process of take-off along the runway, obtain the track traffic situation, obstacles, and other effective information.

During visual landing, the pilot will spend most of the energy on the outside of the cockpit. When height is down to 30 meters, pilots should reference the main ground scenery and special mark to ensure the direction of landing and the location of the ground. In the process, mastery of flight altitude, velocity and aircraft attitude depend entirely on the pilot's visual skills.

Visibility instrument flight, it is possible to avoid the outside world and target characteristics caused by the visual perception ability demands high, but the observation instrument, frequency, focus in order of their regional distribution, and the rationality of the fixation time requirements higher.

Liu zhongqi and his collaborators of Beijing University of Aeronautics and Astronautics have carried on the experimental analysis using eye tracking equipment of eye movement rule, studied the pilot's attention distribution characteristics. Results show that the pilot's attention is mainly focused on three target interest areas of airplane's flat panel display, interest in the target area of the room activities, distribution, and conversion between attention, and the main use of the cognitive mechanism of driver eye movements from top to bottom and allocation of attention [3-5].

Fixation mapping is the "analysis of eye-movement traces" of a given scene. It is often considered a more complex eye tracking measure as it requires more complex analysis of multiple eye tracking metrics, such as mean fixation duration. A fixation map is used in the quantification of the similarity of traces and the degree of coverage by the fixations of a visual stimulus, as shown in figure 2[6]. 


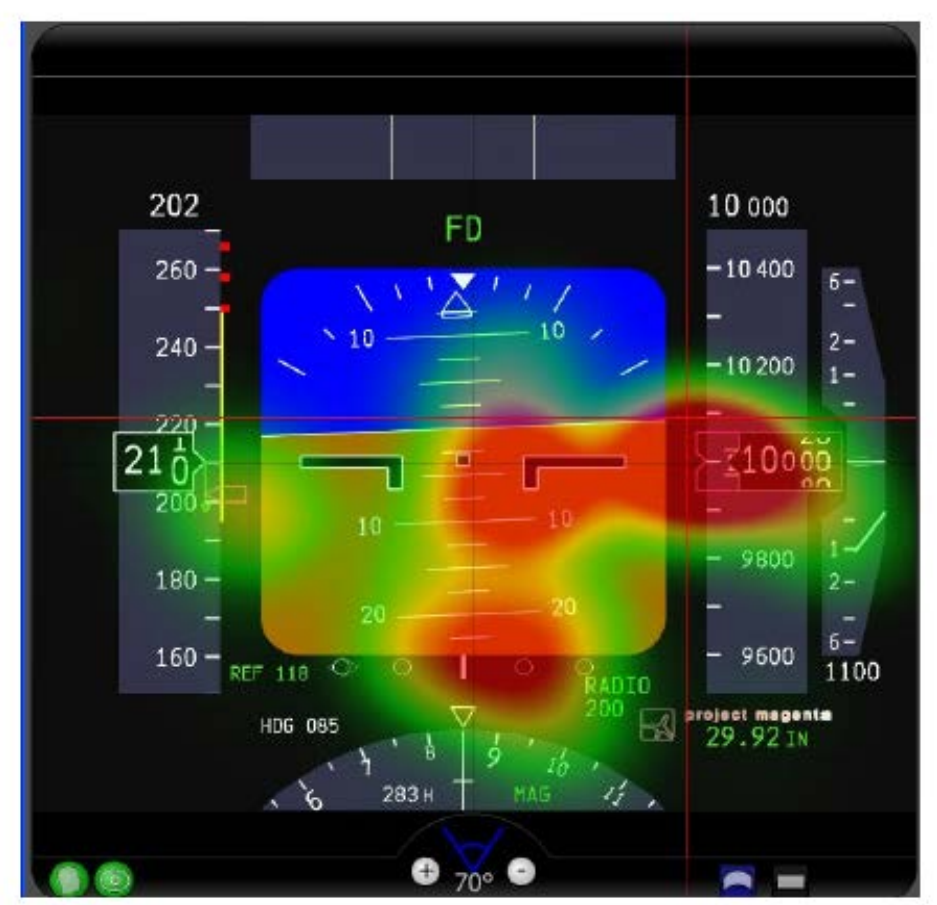

Fig. 2 Example of fixation map

Kim [7] tested the eye movement in the day and night lighting conditions by simulating how the pilot landed his airplane in the process of testing.

NASA carried on a program about Single-Pilot Workload Management in Entry-Level Jets [8]. In this program, eye movements of participants were tracked using a Face Lab ${ }^{\mathrm{TM}} \mathrm{v} 5$ system consisting of non-invasive cameras, IR emitters, and software from Seeing Machines, Inc. Camera set-up and calibration procedures. It is recommended that a system of video and audio time-event markers, called "time hacks," be included in future eye tracking/flight simulator studies.

\section{Summary}

The visual perception is an important link of aircraft control involving human efficiency activities. Research on visual ability and detection and evaluation methods of helps to explore pilot behavior model and the control laws of vehicle, for improving the aircraft cockpit layout and cockpit resource management, improving the flight safety coefficient, improving the efficiency of the flight training, reducing the pilot's workload, etc. All of the related works have important guiding significance to the theory and practice of flight. Although both at home and abroad about the basic rule and method of visual research has made some achievements, but for the increasingly complex environment and the increasing flight information, we also need to carry out the relevant theoretical research and speed up the development of monitoring and testing instrument.

\section{Acknowledgement}

The work on visual inspection is funded by Postdoctoral scientific research funds (2013) of Air Force Aviation University.

\section{References}

[1] Fang ding,Experimental research on driver's visibility dynamic. Dissertation of master degree, (2005).

[2] Liu wei, Yuan xiugan. Research on Human's visual-eye movement system, Chinese Journal of Ergonomics Human Ergonomics, 4 (2000). 
[3] Liu zhongqi, Yuan xiugan, Liu wei, etc. Eye movement analysis in the process of simulated aircraft landing. Journal of Beijing University of Aeronautics and Astronautics, 28(6):703-706(2002).

[4] Liu zhongqi, Yuan xiugan, Liu wei, etc. Based on the eye movement index under the simulated flight mission analysis. China Safety Science Journal, 16(2): 47-51, (2006).

[5] Liu zhongqi, Yuan xiugan, Liu wei, etc. Research on eye movement and cognition in simulation landing. Aerospace Medicine and Medical Engineering, 15(5): 379-380 (2002).

[6] Kyle Kent Edward Ellis. Eye tracking metrics for workload estimation in flight deck operations. Thesis of University of Iowa, 7(2009).

[7] Kim. SA Palmisano, A Ash, et a1. Pilot Gaze andGlideslope Control. Acm Transactions on Applied Perception, 7(3): 1-20(2010).

[8] Barbara K. Burian etal.Single-Pilot Workload Management in Entry-Level Jets , NASA/TM216557(2013). 